\title{
PRIMITIVE OBSTRUCTIONS IN THE COHOMOLOGY OF LOOPSPACES
}

\author{
FRANK WILLIAMS
}

ABstract. Let $X$ and $X^{\prime}$ be $H$-spaces. If $f: \Omega X \rightarrow \Omega X^{\prime}$ is an $H$-map then the obstruction to $f$ being a homotopy-commutative map is a subset $\left\{c_{2}(f)\right\} \subset[\Omega X \wedge$ $\left.\Omega X ; \Omega^{2} X^{\prime}\right]$. In this paper we prove: If $[f]$ is in the image of the composition

$$
\left[P_{k+m} \Omega X ; X^{\prime}\right] \rightarrow\left[\Sigma \Omega X ; X^{\prime}\right] \stackrel{\approx}{\rightrightarrows}\left[\Omega X ; \Omega X^{\prime}\right],
$$

then $\left\{c_{2}(f)\right\}$ is in the image of the composition

$$
\left[P_{k} \Omega X \wedge P_{m} \Omega X ; X^{\prime}\right] \rightarrow\left[\Sigma \Omega X \wedge \Sigma \Omega X ; X^{\prime}\right] \stackrel{\approx}{\approx}\left[\Omega X \wedge \Omega X ; \Omega^{2} X^{\prime}\right] .
$$

Consequently if $\alpha \in H^{n}\left(\Omega X ; Z_{p}\right)$ is an $A_{3}$-class in the sense of Stasheff then each element of $\left\{c_{2}(f)\right\}$ is of the form $\sum c_{i}^{\prime} \otimes c_{i}^{\prime \prime}$ where the $c_{i}^{\prime \prime}$ are primitive.

1. The purpose of this note is to develop a decomposition formula for a certain obstruction class that occurs in the study of $H$-spaces. Let $G$ and $G^{\prime}$ be associative $H$-spaces and $f: G \rightarrow G^{\prime}$ be an $H$-map. For homotopy-commutative $G$ and $G^{\prime}$ we introduced in [6] the notion of $f$ being a $C_{2}$-map. Specifically, if $q$ and $q^{\prime}$ are the commuting homotopies for $G$ and $G^{\prime}$, respectively, and $m$ is a homotopy from $f(x y)$ to $f(x) f(y)$, then $f$ is a $C_{2}$-map provided that there exists a secondary homotopy $r$ : $I^{2} \times G^{2} \rightarrow G^{\prime}$ such that $r(0, t, x, y)=f(q(t, x, y)), r(1, t, x, y)=q^{\prime}(t, f(x), f(y))$, $r(s, 0, x, y)=m(s, x, y)$, and $r(s, 1, x, y)=m(s, y, x)$. The obstruction to the

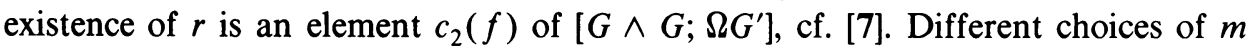
give us a set of obstructions $\left\{c_{2}(f)\right\} \subset\left[G \wedge G ; \Omega G^{\prime}\right]$.

The sets $\left\{c_{2}(f)\right\}$ have proved to be useful in the study of $H$-spaces, see for example $[7,1]$. We shall deal exclusively with the case $G=\Omega X, G^{\prime}=\Omega X^{\prime}$, where $X$ and $X^{\prime}$ are $H$-spaces and $q, q^{\prime}$ are the usual commuting homotopies for the loop multiplications. Now if $G^{\prime}=K\left(Z_{p}, n\right)$, then

$$
\left\{c_{2}(f)\right\} \subset\left[\Omega X \wedge \Omega X ; \Omega K\left(Z_{p}, n\right)\right] \approx H^{n-1}\left(\Omega X \wedge \Omega X ; Z_{p}\right) .
$$

Zabrodsky proved in [7] that if $\alpha \in H^{n}\left(\Omega X, Z_{p}\right)$ is a suspension element then the elements of $\left\{c_{2}(f)\right\}$ may be written in the form $\sum c_{i}^{\prime} \otimes c_{i}^{\prime \prime}$ where the classes $c_{i}^{\prime}$ and $c_{i}^{\prime \prime}$ are also suspensions. One might hope that if $\alpha$ were merely a primitive element then the $c_{i}^{\prime}$ and $c_{i}^{\prime \prime}$ might also turn out to be primitive. This is in general false. In

Received by the editors July 25, 1983.

1980 Mathematics Subject Classification. Primary 55P45, 55S20.

Key words and phrases. $H$-space, homotopy-commutativity, obstruction.

(C1984 American Mathematical Society $0002-9939 / 84 \$ 1.00+\$ .25$ per page 
order to describe what is true in this case, we refer to Stasheff [3], for the definition of the projective spaces of $G$,

$$
\Sigma G=P_{1} G \subset P_{2} G \subset \cdots \subset \bigcup_{k} P_{k} G=P_{\infty} G \sim X .
$$

A map $f: G \rightarrow G^{\prime}$ is called an $A_{k}$-map provided that $\sum f: \sum G \rightarrow \sum G^{\prime}$ extends to a map of filtered spaces $\left\{P_{i} f\right\}:\left\{P_{i} G\right\} \rightarrow\left\{P_{i} G^{\prime}\right\}$. The adjoint of the inclusion $i(1, k)$ : $\sum G \rightarrow P_{k} G$ is an $A_{k}$-map and the adjoint to $i(1, \infty)$ gives the homotopy equivalence of $G$ with $\Omega P_{\infty} G$. Under this equivalence the $A_{\infty}$-maps correspond to the loop maps. Furthermore, the $A_{2}$-maps are simply the $H$-maps. A class $\alpha \in\left[G ; G^{\prime}\right]$ is represented by an $A_{k}$-map if and only if it is in the image of the composition $\left[P_{k} G ; P_{\infty} G^{\prime}\right] \rightarrow$ $\left[\sum G ; P_{\infty} G^{\prime}\right] \stackrel{\widetilde{\rightrightarrows}}{\rightarrow}\left[G ; G^{\prime}\right]$. We shall prove

1.1. THEOREM. If $G$ and $G^{\prime}$ are loop spaces of $H$-spaces and $f: G \rightarrow G^{\prime}$ is an $A_{k+m}$-map, then $\left\{c_{2}(f)\right\}$ is in the image of the composition

$$
\left[P_{k} G \wedge P_{m} G ; P_{\infty} G^{\prime}\right] \rightarrow\left[\Sigma G \wedge \Sigma G ; P_{\infty} G^{\prime}\right] \stackrel{\approx}{\rightarrow}\left[G \wedge G ; \Omega G^{\prime}\right] .
$$

Specializing to the case $G^{\prime}=K\left(Z_{p}, n\right)$, Theorem 1.1 and the Künneth formula give us

1.2. THEOREM. Let $X$ be an $H$-space. If $\alpha \in H^{n}\left(\Omega X ; Z_{p}\right)$ is an $A_{k+m}$-class, then $\left\{c_{2}(\alpha)\right\}$ consists of elements of the form $c_{2}(\alpha)=\sum c_{i}^{\prime} \otimes c_{i}^{\prime \prime}$, where the $c_{i}^{\prime}$ (resp. $\left.c_{i}^{\prime \prime}\right)$ are $A_{k}$-classes (resp. $A_{m}$-classes).

(Since the $A_{\infty}$-classes are the suspension elements, we note that the case $k=m=$ $\infty$ is the above-mentioned result of Zabrodsky.)

In particular, we see that if $\alpha$ is an $A_{3}$-class then we may write elements of $\left\{c_{2}(f)\right\}$ in the form $c_{2}(f)=\sum c_{i}^{\prime} \otimes c_{i}^{\prime \prime}$, where the $c_{i}^{\prime \prime}$ are primitive. The import of Theorem 1.2 is thus that the types of elements that occur in the formula for $c_{2}(f)$ are considerably restricted. This fact is now being applied in the further investigation of the cohomology of finite $H$-spaces (cf. [2]), to extend results originally proved in [5].

2. The proof of Theorem 1.1 consists of the identification of $c_{2}(f)$ with another obstruction, $\theta(f)$, together with some routine diagram-chasing. Let us write $G=\Omega X$, $G^{\prime}=\Omega X^{\prime}$, for $H$-spaces $X$ and $X^{\prime}$. The homotopy equivalence $X \sim P_{\infty} G$ induces a multiplication $\mu$ on $P_{\infty} G$ that may be taken to be filtration-preserving, i.e. $\mu\left(P_{k} G \times P_{m} G\right) \subset P_{k+m} G$ [4]. If $f: G \rightarrow G^{\prime}$ is an $A_{k}$-map, $k \geqslant 2$, we have the (not necessarily commutative) diagram:

$$
\begin{array}{ccc}
\sum G \times \sum G & \stackrel{\mu}{\rightarrow} & P_{2} G \\
\sum f \times \sum f \downarrow & & P_{2} f \downarrow \\
\sum G^{\prime} \times \sum G^{\prime} & \stackrel{\mu^{\prime}}{\rightarrow} & P_{2} G^{\prime} \stackrel{i^{\prime}(2, \infty)}{\rightarrow} P_{\infty} G^{\prime}
\end{array}
$$

The obstruction $\theta(f)$ to a homotopy between $i^{\prime}(2, \infty) \circ \mu^{\prime} \circ\left(\sum f \times \sum f\right)$ and $i^{\prime}(2, \infty) \circ P_{2} f \circ \mu$ is an element of $\left[\sum G \wedge \sum G ; P_{\infty} G^{\prime}\right]$. The next proposition relates $\theta(f)$ to the question at hand. 
2.1. Proposition. Under the isomorphism $\left[\Sigma G \wedge \sum G ; P_{\infty} G\right] \approx\left[G \wedge G ; \Omega G^{\prime}\right], \theta \cdot(f)$ goes to $c_{2}(f)$.

PROOF. This proposition is essentially a version of the adjoint relationship between Whitehead products and Samelson products. It follows from carefully depicting the maps and homotopies that are involved.

Theorem 1.1 now follows from

2.2. Proposition. If $f: G \rightarrow G^{\prime}$ is an $A_{k+m}$-map, then $\theta(f)$ is in the image of

$$
(i(1, k) \wedge i(1, m))^{*}:\left[P_{k} G \wedge P_{m} G ; P_{\infty} G^{\prime}\right] \rightarrow\left[\sum G \wedge \sum G ; P_{\infty} G\right] .
$$

Proof. By definition

$$
\theta(f)=i^{\prime}(2, \infty) \circ \mu^{\prime} \circ\left(\sum f \times \sum f\right)-i^{\prime}(2, \infty) \circ P_{2} f \circ \mu .
$$

By the commutative diagram

$$
\begin{array}{cc}
\sum G \times \sum G \stackrel{i(1, k) \times i(1, m)}{\longrightarrow} P_{k} G \times P_{m} G \\
\downarrow \sum f \times \sum f & \downarrow P_{k} f \times P_{m} f \\
\sum G^{\prime} \times \sum G^{\prime} \longrightarrow i^{\prime}(1, k) \times i^{\prime}(1, m) & P_{k} G^{\prime} \times P_{m} G^{\prime} \\
\downarrow \mu^{\prime} & \downarrow \\
P_{2} G^{\prime} \longrightarrow \mu^{\prime} \\
& P_{k+m} G^{\prime} \\
& \downarrow \\
& P_{\infty} G^{\prime}(2, k+m)
\end{array}
$$

we see that

$i^{\prime}(2, \infty) \circ \mu^{\prime} \circ\left(\sum f \times \sum f\right) \sim i^{\prime}(k+m, \infty) \circ \mu^{\prime} \circ\left(P_{k} f \times P_{m} f\right) \circ(i(1, k) \times i(1, m))$.

And, by the commutative diagram

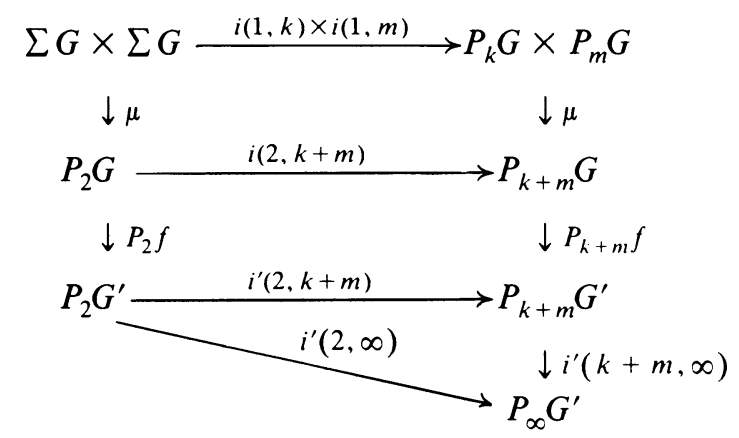

we obtain

$$
i^{\prime}(2, \infty) \circ P_{2} f \circ \mu \sim i^{\prime}(k+m) \circ P_{k+m} f \circ \mu \circ(i(1, k) \times i(1, m)) .
$$

So

$$
\begin{aligned}
\theta(f)= & {\left[i^{\prime}(k+m, \infty) \circ \mu^{\prime} \circ\left(P_{k} f \times P_{m} f\right)-i^{\prime}(k+m, \infty) \circ P_{k+m} f \circ \mu\right] } \\
& \circ(i(1, k) \times 1(1, m)) .
\end{aligned}
$$




\section{REFERENCES}

1. J. Lin, Two torsion and the loop space conjecture, Ann. of Math. (2) 115 (1982), 35-91.

2. A A seven-connected finite $H$-space is fourteen-connected (to appear).

3. J. Stasheff, H-spaces from a homotopy point of view, Lecture Notes in Math., vol. 161, Springer-Verlag, Berlin and New York, 1970.

4. M. Sugawara, On the homotopy-commutativity of groups and loop spaces, Mem. Colloq. Sci. Univ. Kyoto Math. Ser. A 33 (1960/61), 257-269.

5. E. Thomas, Steenrod squares and H-spaces, Ann. of Math. (2) 77 (1963), 306-317.

6. F. Williams, Higher homotopy commutativity, Trans. Amer. Math. Soc. 139 (1969), 191-206.

7. A. Zabrodsky, Cohomology operations and homotopy commutative H-spaces, The Steenrod Algebra and its Applications, Lecture Notes in Math., vol. 168, Springer-Verlag, Berlin and New York, 1970, pp. 308-317.

Department of Mathematics, New Mexico State University, Las Cruces, New Mexico 88003 